Mal J Nutr 27(3): 461-471, 2021

\title{
The correlations between gut microbiota of Muslim Thai lactating women and their dietary intake and gut microbiota of breastfed infants
}

\author{
Lukman Azis ${ }^{1}$, Santad Wichienchot ${ }^{2} \&$ Siwaporn Pinkaew ${ }^{1^{*}}$ \\ ${ }^{1}$ Department of Food Science and Nutrition, Faculty of Science and Technology, Prince \\ of Songkla University, Pattani Campus, Pattani, Thailand; ${ }^{2}$ Center of Excellence \\ in Functional Foods and Gastronomy, Faculty of Agro-Industry, Prince of Songkla \\ University, Hat Yai Campus, Hat Yai, Thailand
}

\begin{abstract}
Introduction: Foods and nutrients are essential not only for human health, but also for the balance of gut microbiota. This research aimed to correlate the gut microbiota of lactating women with their food/ nutrient intakes, as well as with their infants' gut microbiota. Methods: A cross-sectional study was conducted in 27 pairs of mothers and their exclusively breastfed infants. For lactating women, the dietary assessment was conducted by 24-hour recall, and food groups were assessed following the Food and Agriculture Organization's guidelines, while nutrient intake was analysed using INMUNCAL V3 programme. Gut microbiota of mothers and infants were measured in stool samples using fluorescent in situ hybridisation technique. Results: It was found that energy intake of mothers was only $66 \%$ of the recommended Thai Dietary Reference Intakes (DRIs). Most micronutrient and dietary fibre intakes were below the Thai DRIs. Vitamin A (VA)-rich fruits and vegetables food group correlated positively with Lactobacillus species (spp). The association between gut microbiota and nutrient intake of lactating women showed that total protein, phosphorus, and VA were positively correlated with Bifidobacterium spp.; while $\beta$-carotene and vitamin $\mathrm{C}$ were also positively correlated with Lactobacillus spp. In contrast, consumption of eggs and calcium correlated negatively with Clostridium spp./ Enterobacter spp. Bifidobacterium spp. and Lactobacillus spp. of lactating women and breastfed infants showed strong correlations. Conclusion: Food and nutrient intakes of lactating women were correlated with their Clostridium spp./Enterobacter spp., Bifidobacterium spp. and Lactobacillus spp. Furthermore, Bifidobacterium spp. and Lactobacillus spp. of mothers and breastfed infants showed strong correlations.
\end{abstract}

Keywords: correlation, exclusively breastfed infants, food group, gut microbiota, lactating women, nutrient intakes

\section{INTRODUCTION}

Formerly, newborn infants were considered to be in a sterile intrauterine environment (Karakochuk et al., 2017), with their first exposure to microbes occurring during breastfeeding. However, recent studies have suggested that the foetus does not reside in a sterile intrauterine environment, since commensal bacteria from the maternal

\footnotetext{
*Corresponding author: Dr Siwaporn Pinkaew

Department of Food Science and Nutrition, Faculty of Science and Technology

Prince of Songkla University, Pattani, Thailand, 94000

Email: siwaporn.p@psu.ac.th; telp: +66 89977 2266, Fax + 6673334603

doi: https://doi.org/10.31246/mjn-2021-0019
} 
gut/bloodstream can enter the amniotic fluid by crossing the placenta (Walker, 2017). In addition, newborns are also exposed to microbes at birth. The first gut microbiota that interact with an infant have significant roles in stimulating the immune system, facilitating maturation of the immune system, programming a healthy immune development, and metabolic programming (Karakochuk et al., 2017).

The bacterial composition of gut microbiota in breastfed infants is closely related to their mothers'. González et al. (2013) found a positive correlation between maternal milk and infant's faecal microbiota for $S$. epidermidis group, analysed by polymerase chain reaction (PCR). There are several reasons why a correlation exists between a lactating woman and her infant's gut microbiota. The maternal gut microbiota may promote microbiota colonisation of the infant gut prior to birth in utero, after delivery, or later through breastfeeding (McDonald \& McCoy, 2019). Oligosaccharides in breast milk promote the growth of Lactobacillus and Bifidobacterium, which dominate the infant gut (Mueller et al., 2015). Maternal microbiota at different body sites and in the gut provide exposure to an infant at the initial stages of life from holding, cuddling and kissing (Farren \& Turner, 2017), supplying viable microbiota.

An association between the gut microbiota and the infant's nutritional status was found for Streptococcus, Clostridium leptum, and Clostridium coccoides, which were significantly increased in infants with malnutrition (González et al., 2013). A recent study has shown that changes in gut microbiota profile among Thai urban children were related to the types of food consumed (Nakayama et al., 2015). The types of food and nutrients consumed in the dishes are primary factors influencing gut microbiota. For example, fermentation of undigested dietary components generates shortchain fatty acids (SCFA) that are essential for the growth of beneficial bacteria in the intestine (Telle-Hansen, Holven \& Ulven, 2018). Polyphenols, fibres, and carbohydrates are dietary factors that can modify the balance of gut microbiota, whereas illness, stress, ageing, bad dietary habits, and lifestyle can cause changes in the gut microbiota (Zhang et al., 2015).

Recently, a study of the interrelation between nutrient/nutritional status and gut microbiota has recognised this as a determinant of human health (Hills et al., 2019). However, there are needs of supporting evidence, especially of the relationship between gut microbiota in lactating women with their diet and nutrient intakes; as well as of the gut microbiota in their exclusively breastfed infants. Our focus was on southern peninsular Thailand, where the culture and eating habits differ from other parts of the country. Thus, this study aimed to correlate the gut microbiota in lactating women with their food/nutrient intakes, and with their exclusively breastfed infants' gut microbiota.

\section{MATERIALS AND METHODS}

\section{Study site and participants}

A descriptive cross-sectional study was conducted in a sample of 27 Thai Muslim lactating women and their exclusively breastfed infants. This study was part of a main study named "the efficacy of vitamin A (VA) fortified rice in lactating Thai women". The details of the study protocol and sample size calculation are described in a previous report (Pinkaew et al., 2021). Briefly, healthy women with single birth, no gestational diseases, in the age range of 20-40 years old, and their exclusively breastfed infants with a birth weight of $>2,500 \mathrm{~g}$, were recruited for the study from January 2017 to 
February 2018. The study site was located in the Muang district of Pattani province, in southern Thailand.

Before recruitment, details of the study protocol were explained to the potential participants. Informed consent was obtained from the participants, as well as on behalf of their infants.

\section{Data collection}

\section{Dietary intake}

Dietary intakes of the lactating women were assessed by 24-hour recall for three non-consecutive days (two weekdays and one weekend). The data consisted of food items, types of food, food components, cooking method, and the amount (weight/size) of the consumed portion. The data were analysed by INMUCAL V3 programme (Mahidol University, Thailand) for nutrient intakes and reported in \% Thai Dietary Reference Intake (DRI).

In addition to that, data of food items from the 24-hour recall were also grouped. The nine food groups assessed were according to the Food and Agriculture Organization (FAO) guidelines (FAO, 2011). The amounts consumed for (1) starchy staples, (2) dark green leafy vegetables, (3) other VArich fruits and vegetables, (4) other fruits and vegetables, (5) organ meat, (6) meat and fish, (7) eggs, (8) legumes, nut and seeds, and (9) milk and milk products, were estimated by using the same programme (INMUCAL V3 programme).

\section{Gut microbiota determination}

Stool samples of lactating women and their breastfed infants were collected during the first month of breastfeeding. At the time of stool sample collection, the participants were informed about instructions that must be followed on collecting the stool samples. Stool samples of both mother and infant had to be collected on the same day by using a sterile plastic teaspoon and placed into a sterile small plastic box. Then they were brought to the laboratory and kept in a freezer at $-80^{\circ} \mathrm{C}$ for gut microbiota analysis.

All stool samples were analysed specifically for the bacteria Bifidobacterium species (spp.) and Lactobacillus spp. as representatives of beneficial bacteria, and Clostridium spp./Enterobacter spp. as representative of pathogenic bacteria, using DNA probes (Bif164, Lab158, Chris150) (Lucigen, Wisconsin, USA). The fluorescent in situ hybridisation (FISH) technique was used to measure the gut bacteria in stool samples following laboratory protocols of the Nutraceutical and Functional Laboratory (Faculty of Agroindustry, Prince of Songkla University, Hatyai, Thailand) (Plongbunjong et al., 2017). Data were reported as $\log _{10}$ cell/g faecal sample.

\section{Ethical clearance}

The study protocol was approved by the Research Ethics Committee for Science, Technology and Health Science, Prince of Songkla University, Pattani campus (ERC No. psu.pn.1-005/59). The protocol was registered with ClinicalTrials.gov (ID: NCT03056625).

\section{Statistical analysis}

The gut microbiota enumeration by FISH technique was presented as $\log _{10}$ cell/g faecal sample. Data on nutrient intakes were analysed with INMUCAL V-3 programme (Mahidol University, Thailand) and were presented as \% Thai DRI. Data were normally distributed as checked by Shapiro-Wilk test $(p>0.05)$ and were expressed as mean \pm standard deviation $(S D)$. The correlation between gut microbiota in lactating women versus nutrient intakes, amount of food consumption (nine groups), and gut microbiota of infants were assessed 
using Pearson's correlation. All data were statistically analysed with $\mathrm{R}$ programme i386 version 3.5.2.

\section{RESULTS}

The average age of mothers and infants were 29.2 years old and one month old, respectively. All infants were exclusively breastfed, and $70.4 \%$ were born via vaginal delivery. All of the participants were Muslim. The average Bifidobacria spp., Lactobacillus spp., and Clostridium spp./Enterobacter spp. in lactating women were $9.70 \pm 0.28,9.75 \pm 0.36$, and $9.76 \pm 0.42 \log _{10}$ cell/g faecal sample, respectively. For infants, the average Bifidobacria spp., Lactobacillus spp., and Clostridium spp./Enterobacter spp. were $9.82 \pm 0.35,9.71 \pm 0.36$, and $9.70 \pm 0.60$ $\log _{10}$ cell/g faecal sample, respectively.

\section{Dietary intake of lactating women}

Food group consumption of lactating women

The amounts of daily consumption (per day) for the nine groups of food, as presented in Table 1, were assessed following the FAO guidelines (FAO, 2011). A total of $642 \mathrm{~g}$ of starchy staple food was consumed daily, and this was the main food group consumed by lactating women. This was followed by other fruits and vegetables (117.8 g), milk and milk products (113.8 g), and meat and fish (112.0 g). Other VA-rich fruits and vegetables group had the lowest (11.5 g) intake.

\section{Nutrient intakes of lactating women}

On average, the energy intake of the participants was $1,476 \mathrm{kcal} /$ day and this only accounted for $66 \%$ of the Thai DRI. The energy distribution of carbohydrate:protein:fat was in the normal range (64:15:21). Figure 1 showed that both the energy and protein intakes of participants were below the Thai DRIs and estimated average requirement (EAR $\approx 80 \%$ of Thai DRIs).

Most of the micronutrients (Figure 1) and dietary fibre consumed were below the Thai DRIs. Daily VA and iron consumption, which are essential for lactating women, were found to be deficient compared to the recommendation (14\% and $17 \%$ of Thai DRIs, respectively). The consumption of other important micronutrients by

Table 1. The average consumption of each food group by lactating women (g/day)

\begin{tabular}{|c|c|}
\hline Food group & Total $(n=27)$ \\
\hline Starchy staples ${ }^{\dagger}(\mathrm{g})$ & $642.0 \pm 217.3$ \\
\hline Dark green leafy vegetables $(g)$ & $13.1 \pm 2.6$ \\
\hline Other vitamin A-rich fruits and vegetables ${ }^{\ddagger}(\mathrm{g})$ & $11.5 \pm 4.3$ \\
\hline Other fruits and vegetables ${ }^{\S}(\mathrm{g})$ & $117.8 \pm 50.8$ \\
\hline Organ meat $(g)$ & $41.8 \pm 10.4$ \\
\hline Meat and fish" (g) & $112.0 \pm 41.4$ \\
\hline Eggs (g) & $36.8 \pm 9.7$ \\
\hline Legumes, nuts and seeds (g) & $18.9 \pm 3.0$ \\
\hline Milk and milk products (g) & $113.8 \pm 28.8$ \\
\hline
\end{tabular}

${ }^{\dagger}$ The starchy staples food group is a combination of cereals and white roots and tubers.

₹The other VA-rich fruits and vegetables group is a combination of VA-rich vegetables and tubers and VA-rich fruits (e.g. the plants with red, yellow, orange colour).

sThe other fruits and vegetables group is a combination of other fruits and other vegetables (excluding dark green leafy vegetables and other vitamin A-rich fruits and vegetables ${ }^{\ddagger}$ ).

TThe meat group is a combination of meat and fish. 


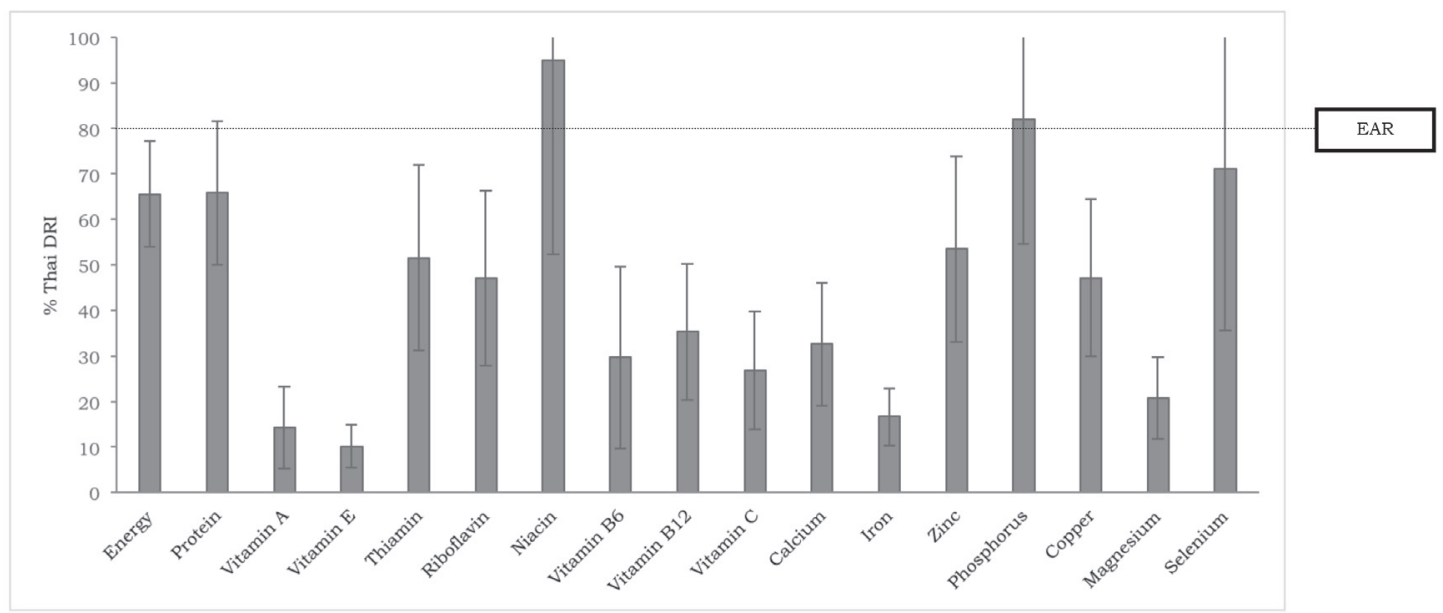

Figure 1. The nutrient intakes of lactating women relative to Thai DRIs.

The dotted line stands for EAR=estimated average requirement (IOM, Food and Nutrition Board, 1998)

lactating women, such as calcium, zinc, thiamine, and vitamin $\mathrm{C}$, were also below the EARs $(33 \%, 53 \%, 52 \%$, and $27 \%$ of Thai DRIs). The intake of dietary fibre was only $6.8 \mathrm{~g} /$ day compared to the recommended daily intake of $25 \mathrm{~g} /$ day. Phosphorus and niacin were the only two nutrient intakes higher than the EARs (> $80 \%$ of Thai DRIs.)
Correlation of gut bacteria and dietary intake of lactating women Correlation of gut bacteria and food group intakes of lactating women Table 2 shows the associations between the group of foods consumed by lactating women and Bifidobacterium spp., Lactobacillus spp., and Clostridium spp./Enterobacter spp. Among the nine

Table 2. Correlation coefficients (cc) of food group consumption and gut bacteria in lactating women

\begin{tabular}{lccc}
\hline & \multicolumn{3}{c}{ Correlation coefficients } \\
\cline { 2 - 4 } Food group & $\begin{array}{c}\text { Bifidobacterium } \\
\text { spp. }\end{array}$ & $\begin{array}{c}\text { Lactobacillus } \\
\text { spp. }\end{array}$ & $\begin{array}{c}\text { Clostridium spp./ } \\
\text { Enterobacter spp. }\end{array}$ \\
\hline Starchy staples & -0.15 & 0.31 & 0.09 \\
Dark green leafy vegetables & 0.23 & -0.05 & -0.19 \\
Other vitamin A rich fruits and & -0.03 & $0.47^{*}$ & -0.03 \\
vegetables & -0.16 & -0.26 & -0.23 \\
Other fruits and vegetables & -0.18 & 0.03 & -0.27 \\
Organ meat & 0.05 & -0.15 & -0.14 \\
Meat and fish & -0.03 & 0.07 & $-0.45^{*}$ \\
Eggs & 0.13 & 0.004 & 0.32 \\
Legumes, nut and seeds & 0.13 & -0.15 & 0.11 \\
Milk and milk products & &
\end{tabular}

"correlation (Pearson's correlation) is significant at the 0.05 level (2-tailed) 
groups of food consumed, Lactobacillus showed a positive correlation with "other VA-rich fruits and vegetables". However, lactating women with high egg consumption showed a negative correlation with Clostridium spp./ Enterobacter spp.

Correlation of gut bacteria and nutrient intakes of lactating women

The correlations of Bifidobacterium spp., Lactobacillus spp., and Clostridium spp./Enterobacter spp. and nutrient intakes are shown in Table 3. Proteins, protein from animal, phosphorus, and VA were positively correlated with
Bifidobacterium, while $\beta$-carotene and vitamin $\mathrm{C}$ were positively correlated with Lactobacillus spp. However, Clostridium spp./Enterobacter spp. was negatively correlated with calcium.

\section{Correlation of gut bacteria between lactating women and their breastfed infants}

As shown in Table 4, Bifidobacterium spp. and Lactobacillus spp. of lactating women and infants were positively correlated. However, no correlation was detected for Clostridium spp./ Enterobacter spp.

Table 3. Correlation coefficients (cc) of nutrient intakes with gut bacteria in lactating women

\begin{tabular}{lccc}
\hline \multirow{2}{*}{ Nutrient } & \multicolumn{3}{c}{ Correlation coefficients } \\
\cline { 2 - 4 } & Bifidobacterium spp. & Lactobacillus spp. & $\begin{array}{c}\text { Clostridium spp/ } \\
\text { Enterobacter spp. }\end{array}$ \\
\hline Energy & 0.21 & 0.06 & 0.28 \\
Carbohydrate & 0.31 & 0.18 & 0.39 \\
Protein & $0.55^{*}$ & 0.27 & 0.17 \\
Protein-animal & $0.55^{*}$ & 0.22 & 0.23 \\
Protein-vegetable & 0.39 & 0.31 & 0.32 \\
Fat & -0.19 & -0.21 & -0.35 \\
Calcium & -0.20 & 0.15 & $-0.50^{*}$ \\
Phosphorus & $0.50^{*}$ & 0.19 & 0.02 \\
Iron & 0.31 & -0.08 & 0.07 \\
Copper & -0.07 & -0.15 & 0.28 \\
Magnesium & 0.27 & 0.32 & -0.12 \\
Selenium & 0.33 & 0.15 & 0.31 \\
Zinc & 0.22 & -0.10 & -0.06 \\
Vitamin A & $0.50^{*}$ & 0.37 & 0.27 \\
Retinol & -0.21 & 0.06 & -0.07 \\
Beta-carotene & -0.32 & $0.49^{*}$ & -0.16 \\
Thiamin & 0.15 & 0.01 & 0.01 \\
Riboflavin & -0.08 & -0.37 & -0.39 \\
Vitamin B6 & 0.34 & -0.14 & 0.01 \\
Vitamin B12 & 0.25 & -0.10 & -0.09 \\
Vitamin C & -0.29 & $0.47^{*}$ & 0.24 \\
Niacin & 0.28 & 0.20 & 0.08 \\
Vitamin E & -0.16 & -0.40 & -0.29 \\
Dietary fibre & 0.13 & -0.07 & 0.04 \\
\hline
\end{tabular}

"correlation (Pearson's correlation) is significant at the 0.05 level (2-tailed) 
Table 4. Correlation coefficients (cc) of gut bacteria in lactating women and in their infants

\begin{tabular}{lccc}
\hline \multirow{2}{*}{ Bacteria } & \multicolumn{3}{c}{ Lactating women bacteria } \\
\cline { 2 - 4 } & $\begin{array}{c}\text { Bifidobacterium } \\
(\mathrm{cc})\end{array}$ & $\begin{array}{c}\text { Lactobacillus } \\
(\mathrm{cc})\end{array}$ & $\begin{array}{c}\text { Clostridium/ } \\
\text { Enterobacter }(c c)\end{array}$ \\
\hline Infant bacteria & $0.53^{* *}$ & $0.47^{*}$ & 0.36 \\
Bifidobacterium (cc) & $0.62^{* *}$ & $0.51^{*}$ & 0.29 \\
Lactobacillus (cc) & 0.17 & 0.40 & 0.01 \\
Clostridium/Enterobacter (cc) & &
\end{tabular}

"correlation (Pearson's correlation) is significant at the 0.05 level (2-tailed)

"correlation (Pearson's correlation) is significant at the 0.01 level (2-tailed)

cc, correlation coefficient

\section{DISCUSSION}

Data on the average food consumption (nine food groups) and nutrient intakes of mothers revealed that lactating women in this study area may have a risk of undernutrition, especially a lack of vitamin A. This is because most of the nutrient and energy intakes of lactating women were below the Thai DRIs and EARs. The average energy intake was only $1,476 \mathrm{kcal} / \mathrm{day}$, which accounted for $66 \%$ of the Thai DRI, while the average VA intake of participants was only $14 \%$ of the Thai DRI. The percentage of VA was lower than in other developing countries such as Indonesia and China. VA intake of lactating women in Indonesia was $64 \%$ of Angka Kecukupan Gizi (AKG), while China had $100.1 \%$ of its Chinese Recommended Nutrient Intake (RNI) (Chen et al., 2012; Madanijah et al., 2016). The main study had revealed that almost $50 \%$ of subjects had VA deficiency based on total VA liver reserve (Pinkaew et al., 2021), while the average body mass index (BMI) was 22.5 \pm 3.9 $\mathrm{kg} / \mathrm{m}^{2}$ (Azis, Pinkaew \& Wichienchot, 2021). However, according to Swalarz et al. (2018), BMI is not the best indicator of malnutrition, because malnutrition already occurs much earlier and there is no cut-off for lactating women. The subjects had low energy intake compared to the requirement for lactating women, mainly due to low socio-economic status with an average family income of USD500 per month to support around five people in a family (Azis et al., 2021). Moreover, food taboos of the Muslim people in the study area, which only allowed the consumption of rice and grilled or fried fish during the first period of lactation (30 days - three months) may have further contributed to the lack in energy intake.

Among the nine food groups consumed by lactating women (FAO, 2011), rice was the main starchy staple with the highest amount of consumption (642 g/day). This was the food group that contributed the main portion of calories and other nutrients to these lactating women.

The correlations between gut bacteria in lactating women and food groups showed that the "other VA-rich fruits and vegetables" was positively correlated with Lactobacillus spp. A study showed that Lactobacilli are able to use foods as their carbon source to support their growth and maintain the environmental pH (Walter, 2008). Previous in vivo and in vitro studies have found an interrelationship between Lactobacillus spp. and VA group of foods (Lee \& Ko, 2016). However, the underlying mechanism has not been clearly elaborated. Cell differentiation and maintenance of healthy skin, mucous membranes, and soft tissues, 
are the major functions of VA (Huang et al., 2018). Therefore, VA could potentially help in promoting differentiation of healthy villi in the gastrointestinal tract. Moreover, fruits and vegetables are known as important sources of carbohydrates and prebiotics, such as fructooligosaccharides, oligofructose, inulin, galactose, and xylose, which contain oligosaccharides as energy sources used by the gut microbiota (Azad et al., 2018).

The results revealed that Bifidobacterium spp. was positively associated with total protein, protein from animal sources, phosphorus, and VA intake. Garcia-Mantrana et al. (2018) reported that protein-rich foods have significantly high bifidobacteria counts and significant positive correlations with various groups of butyrate-producing bacteria within the faecal microbial composition, along with higher concentration of SCFA. In haemodialysis patients, Bifidobacteriaceae was positively correlated with phosphorus. Bifidobacterium spp. may regulate the absorption of phosphate in the intestine and use it as one of their energy sources (Miao et al., 2018).

Lactobacillus spp. was positively correlated with $\beta$-carotene and vitamin C. $\beta$-carotene is a good source of VA. Therefore, this could support our finding of a positive correlation between "other VA-rich fruits and vegetables" and Lactobacillus spp. A prior study found that beta-carotene as pro-VA carotenoid promoted Bifidobacteria and Lactobacillus via fermentation of the substances bound with $\beta$-carotene in the food matrix (O'Callaghan \& van Sinderen, 2016). The proposed mechanism of the correlation between vitamin $\mathrm{C}$ and Lactobacillus spp. is that vitamin C might stimulate the growth of lactobacilli by increasing the acidity of the medium, as probiotics prefer an acidic gastric environment (Stadler \& Viernstein, 2003). Lactobacilli of intestinal origin are considered intrinsically resistant to acidic environments and are often employed in fermented foods as probiotics (Corcoran et al., 2005). On the other hand, calcium was negatively correlated with Clostridium spp./Enterobacter spp. Previously, calcium was also reported to be negatively correlated with Clostridium coccoides and Clostridium leptum in obese mice, which is in line with our finding. Calcium can modulate gut microbiota in a specific and prebiotic manner (Chaplin et al., 2016).

The association between gut microbiota of lactating women and those of their breastfed infants has been confirmed in our Muslim population. Bifidobacterium spp. and Lactobacillus spp. in lactating women and infant pairs were positively correlated. According to Karakochuk et al. (2017), the gut microorganisms in mothers are closely similar with their infants'. This is because of their food pattern, environment, activity, and daily interaction, as well as the mode of delivery. Bifidobacteria species e.g. B. breve, B. longum spp., B. bifidum, B. adolescentis, B. dentium, and B. pseudocatenulatum, which were all identified in infant faeces, were also found in the corresponding milk samples of the mother (Duranti et al., 2017). The transmission of Bifidobacteria and Lactobacillus of lactating women to her infant suggests that human milk is a potential vehicle to facilitate this acquisition.

This is the first study on Thai Muslim population that presented a correlation between gut bacteria and dietary/ nutrient intakes of lactating women, and also of gut bacteria in their exclusively breastfed infants. Our data showed that Lactobacillus spp. of lactating women was positively correlated with VA-rich fruits/vegetables food group 
(providing carotenoids), $\beta$-carotene (major source of carotenoids in the diet), and Lactobacillus spp. of their breastfed infants. Thus, VA/ $\beta$-carotene in the diet of mothers could be a factor promoting the growth of Lactobacillus spp., not only in the mothers themselves, but also in their infants.

A limitation of this current study was the rather small sample size $(27$ pairs of lactating women and breastfed infants) due to the high rate of dropouts, mainly caused by inability to exclusively breastfeed. As a limitation of descriptive cross-sectional study, it was not possible to establish a true cause and effect. Therefore, a longitudinal or experimental study should be conducted to confirm this finding. In addition to that, aside from diet/nutrients, several other factors such as maternal size, mode of delivery, feeding practices, and body contact are also known to affect the gut microbiota of lactating women and infants; these could be addressed by a future study together with nutritional factors.

The findings from this study supported the need for encouraging exclusive breastfeeding for the first six months of a newborn's life, together with giving nutrition education to mothers. These strategies could shape the nutritional status and promote the growth of beneficial bacteria (Lactobacillus spp. and Bifidobacterium spp.) in both mothers and infants.

\section{CONCLUSION}

Based on the dietary assessment, the lactating women in this study area were at risk of undernutrition and rice was consumed as the main staple food. The "VA-rich fruits and vegetables" food group correlated positively with Lactobacillus spp. Egg consumption correlated negatively with Clostridium spp./Enterobacter spp. Regarding associations between gut microbiota and nutrient intakes in lactating women, total protein, protein from animal sources, phosphorus, and VA were positively correlated with Bifidobacterium spp. Beta-carotene and vitamin $\mathrm{C}$ were also positively correlated with Lactobacillus spp., but calcium was negatively correlated with Clostridium spp./Enterobacter spp. Finally, Bifidobacterium spp. and Lactobacillus spp. of lactating women and their breastfed infants showed strong correlations.

\section{Acknowledgements}

Sincere appreciation goes to the participants who consistently carried out exclusive breastfeeding of their infants. Special thanks to the research assistant team and staff members of the healthpromoting hospital for their cooperation. We would like to thank the Research and Development office, Prince of Songkla University and Assoc. Prof. Dr. Seppo Karrila for assistance in manuscript preparation. The research was funded by Halal Food Science Center, Department of Food Science and Nutrition, Faculty of Science and Technology, Prince of Songkla University and Graduate School research funding of the Prince of Songkla University, Thailand.

\section{Authors' contributions}

LA, conducted the dietary assessment, measured gut microbiota enumeration, statistically analysed the data, and drafted the manuscript; SW, designed the research and supervised gut microbiota determination; SP, designed the research, conducted the dietary assessment, and statistically analysed the data. All authors read and approved the final manuscript.

\section{Conflict of interest}

LA, SW and SP have no conflicts of interest.

\section{References}

Azad MAK, Sarker M, Li T \& Yin J (2018). Probiotic species in the modulation of gut microbiota: An overview. BioMed Res Int 2018:1-8. https:// doi.org/10.1155/2018/9478630

Azis L, Pinkaew S \& Wichienchot S (2021). Effect of vitamin a-fortified rice on the gut microbiota of Thai lactating women and their exclusively breastfed infants. Chiang Mai Univ J Nat Sci 20(4):e202 1079-93. 
Chaplin A, Parra P, Laraichi S, Serra F \& Palou A (2016). Calcium supplementation modulates gut microbiota in a prebiotic manner in dietary obese mice. Mol Nutr Food Res 60(2):468-480. https://doi.org/10.1002/mnfr.201500480

Chen H, Wang P, Han Y, Ma J, Troy FA \& Wang B (2012). Evaluation of dietary intake of lactating women in China and its potential impact on the health of mothers and infants. BMC Women's Health 12(18):1-10. https: / / doi. org/10.1186/1472-6874-12-18

Corcoran BM, Stanton C, Fitzgerald GF \& Ross RP (2005). Survival of probiotic lactobacilli in acidic environments is enhanced in the presence of metabolizable sugars. Appl Environ Microbiol 71(6):3060-3067. https://doi.org/10.1128/ AEM.71.6.3060-3067.2005

Duranti S, Lugli GA, Mancabelli L, Armanini F, Turroni F, James K, Ferretti P, Gorfer V, Ferrario C, Milani C, Mangifesta M, Anzalone R, Zolfo M, Viappiani A, Pasolli E, Bariletti I, Canto R, Clementi R, Cologna M, ... Ventura M (2017). Maternal inheritance of bifidobacterial communities and bifidophages in infants through vertical transmission. Microbiome 5(1):1-13. https://doi.org/10.1186/s40168017--0282-6

FAO (2011). Guidelines for measuring household and individual dietary diversity. Kennedy G, Ballard T, and Dop M (eds.). FAO, Rome, Italy. https://www.fao.org/3/i1983e/i1983e.pdf

Farren M \& Turner MJ (2017). Obesity and gestational diabetes. In CD Karakochuk, KC Whitfield, TJ Green, K Kraemer (eds). The Biology of the First 1,000 Days (pp. 145154). CRC Press, New York. https://doi. org/10.1201/9781315152950

Garcia-Mantrana I, Selma-Royo M, Alcantara C \& Collado MC (2018). Shifts on gut microbiota associated to mediterranean diet adherence and specific dietary intakes on general adult population. Front Microbiol 9(890):1-11. https://doi.org/10.3389/fmicb.2018.00890

González R, Mandomando I, Fumadó V, Sacoor C, Macete E, Alonso PL \& Menendez C (2013). Breast milk and gut microbiota in African mothers and infants from an area of high HIV prevalence. PLOS ONE 8(11):1-9. https://doi. org/10.1371/journal.pone.0080299

Hills RD, Pontefract BA, Mishcon HR, Black CA, Sutton SC \& Theberge CR (2019). Gut microbiome: Profound implications for diet and disease. Nutrients 11(7):1-18. https://doi. org/10.3390/nu11071613
Huang Z, Liu Y, Qi G, Brand D \& Zheng SG (2018). Role of vitamin $A$ in the immune system. Clin Med 7(9):1-16. https://doi.org/10.3390/ jcm7090258

IOM, Food and Nutrition Board (1998). Dietary reference intakes: a risk assessment model for establishing upper intake levels for nutrients. Nutr Rev 55(9):1-12.

Karakochuk CD, Whitfield KC, Green TJ \& Kraemer K (2017). The Biology of the First 1,000 Days. https://doi.org/10.1201/9781315152950

Lee H \& Ko GP (2016). Antiviral effect of Vitamin A on norovirus infection via modulation of the gut microbiome. Sci Rep 6(1):1-9. https://doi. org/10.1038/srep25835

Madanijah S, Rimbawan R, Briawan D, Zulaikhah $Z$, Andarwulan N, Nuraida L, Sundjaya, T, Murti L \& Bindels J (2016). Nutritional status of lactating women in Bogor district, Indonesia: cross-sectional dietary intake in three economic quintiles and comparison with pre-pregnant women. Br J Nutr 116(1):S67-S74. https://doi. org/10.1017/S0007114516001306

McDonald B \& McCoy KD (2019). Maternal microbiota in pregnancy and early life. Science 365(6457):984-985. https://doi.org/10.1126/ science.aay0618

Miao YY, Xu CM, Xia M, Zhu HQ \& Chen YQ (2018). Relationship between gut microbiota and phosphorus metabolism in hemodialysis patients: A preliminary exploration. Chin Med J 131(23):2792-2799. https://doi. org/ 10.4103/0366-6999.246059

Mueller NT, Bakacs E, Combellick J, Grigoryan $Z$ \& Dominguez-Bello MG (2015). The infant microbiome development: Mom matters. Trends Mol Med 21(2):109-117. https://doi. org/10.1016/j.molmed.2014.12.002

Nakayama J, Watanabe K, Jiang J, Matsuda K, Chao SH, Haryono P, La-Ongkham O, Sarwoko MA, Sujaya IN, Zhao L, Chen KT, Chen YP, Chiu HH, Hidaka T, Huang NX, Kiyohara C, Kurakawa T, Sakamoto N, Sonomoto K, ... Lee YK (2015). Diversity in gut bacterial community of school-age children in Asia. Sci Rep 5(8397):1-12. https://doi.org/10.1038/ srep08397

O'Callaghan A \& van Sinderen D (2016). Bifidobacteria and their role as members of the human gut microbiota. Front Microbiol 7(925):1-23. https: / / doi.org/ 10.3389/

fmicb.2016.00925 
Pinkaew S, Udomkesmalee E, Davis CR \& Tanumihardjo SA (2021). Vitamin A-fortified rice increases total body vitamin A stores in lactating Thai women measured by retinol isotope dilution: a double-blind, randomized, controlled trial. Am J Clin Nutr https://doi. org/10.1093/ajcn/nqaa418

Plongbunjong V, Graidist P, Knudsen KEB \& Wichienchot S (2017). Isomaltooligosaccharide synthesised from rice starch and its prebiotic properties in vitro. Int $J$ Food Sci Technol 52(12):2589-2595. https://doi.org/10.1111/ ijfs. 13545

Stadler M \& Viernstein H (2003). Optimization of a formulation containing viable lactic acid bacteria. Int $J$ Pharm 256(1-2):117122 . 5173(03)00068-1

Swalarz M, Swalarz G, Juszczak K, Maciukiewicz P, Czurak K, Matuszewski M, Gajewska D, Słojewski M, Bogacki R, Bryniarski P, Paradysz A, Kadłubowski M, Drewa T \& Genge E (2018). Correlation between malnutrition, body mass index and complications in patients with urinary bladder cancer who underwent radical cystectomy. Adv Clin Exp Med 27(8):11411147. https://doi.org/10.17219/acem/89863
Telle-Hansen VH, Holven KB \& Ulven SM (2018). Impact of a healthy dietary pattern on gut microbiota and systemic inflammation in humans. Nutrients 10(11):1-14. https://doi. org/10.3390/nu10111783

Walker WA (2017). The importance of appropriate initial bacterial colonization of the intestine in newborn, child, and adult health. Pediatr Res 82(3):387-395. https://doi.org/10.1038/ pr.2017.111

Walter $J$ (2008). Ecological role of lactobacilli in the gastrointestinal tract: Implications for fundamental and biomedical research. Appl Environ Microbiol 74(16):4985-4996. https:// doi.org/ 10.1128/AEM.00753-08

Zhang YJ, Li S, Gan RY, Zhou T, Xu DP \& Li HB (2015). Impacts of gut bacteria on human health and diseases. Int J Mol Sci 16(4):74937519. https://doi.org/10.3390/ijms 16047493 